Advances in Complex Systems

(C) World Scientific Publishing Company

\title{
A MODEL OF LARGE-SCALE PROTEOME EVOLUTION
}

\author{
Ricard V. Solé ${ }^{1,2,3}$, Romualdo Pastor-Satorras ${ }^{1}$, Eric Smith ${ }^{2}$, and Thomas B. Kepler ${ }^{2}$ \\ ${ }^{1}$ ICREA-Complex Systems Research Group, FEN \\ Universitat Politècnica de Catalunya, Campus Nord B4, 08034 Barcelona, Spain \\ ${ }^{2}$ Santa Fe Institute, 1399 Hyde Park Road, New Mexico 87501, USA \\ ${ }^{3}$ NASA-associated Astrobiology Institute, INTA/CSIC, Carr. del Ajalvir Km4, Madrid, Spain
}

\begin{abstract}
The next step in the understanding of the genome organization, after the determination of complete sequences, involves proteomics. The proteome includes the whole set of protein-protein interactions, and two recent independent studies have shown that its topology displays a number of surprising features shared by other complex networks, both natural and artificial. In order to understand the origins of this topology and its evolutionary implications, we present a simple model of proteome evolution that is able to reproduce many of the observed statistical regularities reported from the analysis of the yeast proteome. Our results suggest that the observed patterns can be explained by a process of gene duplication and diversification that would evolve proteome networks under a selection pressure, favoring robustness against failure of its individual components.
\end{abstract}

Keywords: Genomics, proteomics, gene duplication, small-world, networks

\section{Introduction}

The genome is one of the most fascinating examples of the importance of emergence from network interactions. The recent sequencing of the human genome [23, 38 revealed some unexpected features and confirmed that "the sequence is only the first level of understanding of the genome" [38]. The next fundamental step beyond the determination of the genome sequence involves the study of the properties of the proteins the genes encode, as well as their interactions [12]. Protein interactions play a key role at many different levels and its failure can lead to cell malfunction or even apoptosis, in some cases triggering neoplastic transformation. This is the case, for example, of the feedback loop between two well-known proteins, MDM2 and p53: in some types of cancers, amplification of the first (an oncoprotein) leads to the inactivation of p53, a tumor-suppressor gene that is central in the control of the cell cycle and death 47 .

Understanding the specific details of protein-protein interactions is an essential part of our understanding of the proteome, but a complementary approach is provided by the observation that network-like effects play also a key role. Using 
again p53 as an example, this gene is actually involved in a large number of interaction pathways dealing with cell signaling, the maintenance of genetic stability, or the induction of cellular differentiation 39]. The failure in p53, as when a highly connected node in the Internet breaks [i], has severe consequences.

Additional insight is provided by the observation that in many cases the total suppression of a given gene in a given organism leads to a small phenotypic effect or even no effect at all [32,41]. These observations support the idea that, although some genes might play a key role and their suppression is lethal, many others can be replaced in their function by some redundancy implicit in the network of interacting proteins.

Protein-protein interaction maps have been studied, at different levels, in a variety of organisms including viruses [5. 13, 25], prokaryotes [31, yeast 18, and multicellular organisms such as C. elegans [14]. Most previous studies have used the so-called two-hybrid assay [14] based on the properties of site-specific transcriptional activators. Although differences exist between different two-hybrid projects [16] the statistical patterns used in our study are robust.

Recent studies have revealed a surprising result: the protein-protein interaction networks in the yeast Saccharomyces cerevisiae share some universal features with other complex networks [35]. These studies actually offer the first global view of the proteome map. These are very heterogeneous networks: The probability $P(k)$ that a given protein interacts with other $k$ proteins is given by a power law, i.e. $P(k) \sim k^{-\gamma}$ with $\gamma \approx 2.5$ (see figure $\mathbb{1}$ ), with a sharp cut-off for large $k$. This distribution is thus very different from the Poissonian shape expected from a simple (Erdos-Renyi) random graph [6:22]. Additionally, these maps also display the socalled small-world (SW) effect: they are highly clustered (i.e. each node has a welldefined neighborhood of "close" nodes) but the minimum distance between any two randomly chosen nodes in the graph is short, a characteristic feature of random graphs 445].

As shown in previous studies [1] this type of networks is extremely robust against random node removal but also very fragile when removal is performed selectively on the most connected nodes. SW networks appear to be present in a wide range of systems, including artificial ones [4, 2, 10,29] and also in neural networks [45, 34, metabolic pathways [8,20,43] (see also [28]), even in human language organization [9]. The implications of these topologies are enormous also for our understanding of epidemics 30,24].

The experimental observations on the proteome map can be summarized as follows:

(1) The proteome map is a sparse graph, with a small average number of links per protein. In 42] an average connectivity $\bar{K} \sim 1.9-2.3$ was reported for the proteome map of $S$. cerevisiae. This observation is also consistent with the study of the global organization of the $E$. coli gene network from available information on transcriptional regulation [36]. 

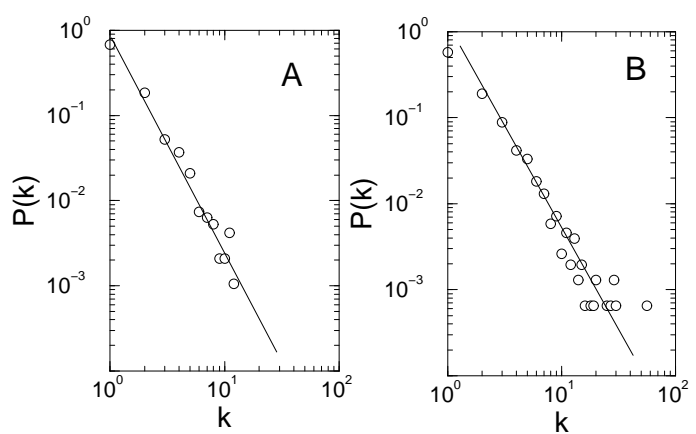

Fig. 1. Degree distributions for two different data sets from the Yeast proteome: A: Ref. 442]; B: Ref. [19]. Both distributions display scaling behavior in their degree distribution $P(k)$, i.e. $P(k) \sim k^{-\gamma}$, a sharp cut-off for large $k$ and very small average connectivities: $\bar{K}_{A}=1.83$ (total graph) and $\bar{K}_{B}=2.3$ (giant component), respectively. The slopes are $\gamma_{A} \approx 2.5 \pm 0.15$ and $\gamma_{B} \approx 2.4 \pm 0.21$.

(2) It exhibits a SW pattern, different from the properties displayed by purely random (Poissonian) graphs.

(3) The degree distribution of links follows a power-law with a well-defined cut-off. To be more precise, Jeong et al. [19] reported a functional form for the degree distribution of $S$. cerevisiae

$$
P(k) \simeq\left(k_{0}+k\right)^{-\gamma} e^{-k / k_{c}} .
$$

A best fit of the real data to this form yields a degree exponent $\gamma \approx 2.5$ and a cut-off $k_{c} \approx 20$. This could have adaptive significance as a source of robustness against mutations.

In this paper we present a model of proteome evolution aimed at capturing the main properties exhibited by protein networks. The basic ingredients of the model are gene duplication plus re-wiring of the protein interactions, two elements known to be the essential driving forces in genome evolution [27]. The model does not include functionality or dynamics of the proteins involved, but it is a topologicallybased approximation to the overall features of the proteome graph and intends to capture some of the generic features of proteome evolution.

During the completion of this work we became aware of a paper by Vázquez et al., Ref. 37, in which a related model of proteome evolution, showing multifractal connectivity properties, is described and analyzed.

\section{Proteome growth model}

Here we restrict our rules to single-gene duplications, which occur in most cases due to unequal crossover [27], plus re-wiring. Multiple duplications should be considered in future extensions of these models: molecular evidence shows that even wholegenome duplications have actually occurred in S. cerevisiae [46] (see also Ref. [40]). 
(a)

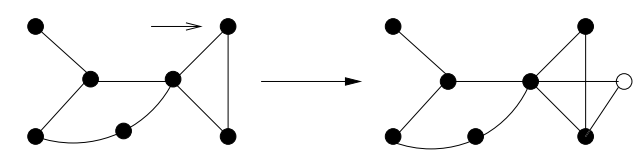

(b)

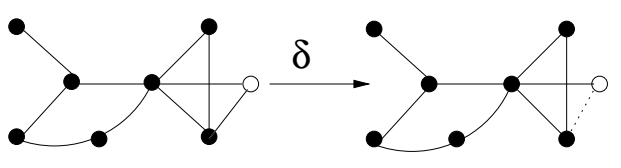

(c)

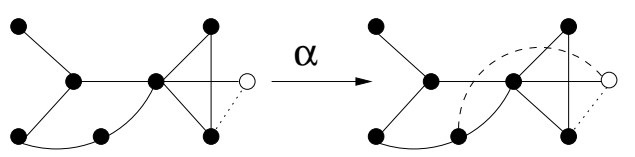

Fig. 2. Growing network by duplication of nodes. First (a) duplication occurs after randomly selecting a node (arrow). The links from the newly created node (white) now can experience deletion (b) and new links can be created (c); these events occur with probabilities $\delta$ and $\alpha$, respectively.

Re-wiring has also been used in dynamical models of the evolution of robustness in complex organisms [7].

It is worth mentioning that the study of metabolic networks provides some support to the rule of preferential attachment 1 国 as a candidate mechanism to explain the origins of the scale-free topology. Scale-free graphs are easily obtained in a growing network provided that the links to new nodes are made preferentially from nodes that already have many links. A direct consequence is that vertices with many connections are those that have been incorporated early. This seems to be plausible in the early history of metabolic nets, and this view is supported by some available evidence [43]. A similar argument can be made with proteome maps, since there are strong connections between the evolution of metabolic pathways and genome evolution, and other scenarios have also been proposed, including optimization [11]. Here we do not consider preferential attachment rules, although future studies should explore the possible contributions of different mechanisms to the evolution of network biocomplexity. In this context, new integrated analyses of cellular pathways using microarrays and quantitative proteomics [17] will help to obtain a more detailed picture of how these networks are organized.

The proteome graph at any given step $t$ (i.e. after $t$ duplications) will be indicated as $\Omega_{p}(t)$. The rules of the model, summarized in figure 2 , are implemented as follows. Each time step: (a) one node in the graph is randomly chosen and duplicated; (b) the links emerging from the new generated node are removed with probability $\delta$; (c) finally, new links (not previously present) can be created between the new node and all the rest of the nodes with probability $\alpha$. Step (a) implements gene duplication, in which both the original and the replicated proteins retain the same structural properties and, consequently, the same set of interactions. The rewiring 
steps (b) and (c) implement the possible mutations of the replicated gene, which translate into the deletion and addition of interactions, with different probabilities.

Since we have two free parameters, we should first constrain their possible values by using the available empirical data. As a first step, we can estimate the asymptotic average connectivity exhibited by the model in a mean-field approximation (see also Ref. [37]). Let us indicate by $\bar{K}_{N}$ the average connectivity of the system when it is composed by $N$ nodes. It is not difficult to see that the increase in the average connectivity after one iteration step of the model is proportional to

$$
\frac{d \bar{K}_{N}}{d N} \simeq \bar{K}_{N+1}-\bar{K}_{N}=\frac{1}{N}\left[\bar{K}_{N}-2 \delta \bar{K}_{N}+2 \alpha\left(N-\bar{K}_{N}\right)\right] .
$$

The first term accounts for the duplication of one node, the second represents the average elimination of $\delta \bar{K}_{N}$ links emanating from the new node, and the last term represents the addition of $\alpha\left(N-\bar{K}_{N}\right)$ new connections pointing to the new node. Eq. (2.1) is a linear equation which easily solved, yielding

$$
\bar{K}_{N}=\frac{\alpha N}{\alpha+\delta}+\left(\bar{K}_{1}-\frac{\alpha}{\alpha+\delta}\right) N^{\Gamma},
$$

where $\Gamma=1-2 \alpha-2 \delta$ and $\bar{K}_{1}$ is the initial average connectivity of the system. This solution leads to an increasing connectivity through time. In order to have a finite $\bar{K}$ in the limit of large $N$, we must impose the condition $\alpha=\beta / N$, where $\beta$ is a constant. That is, the rate of addition of new links (the establishment of new viable interactions between proteins) is inversely proportional to the network size, and thus much smaller than the deletion rate $\delta$, in agreement with the rates observed in [42]. In this case, for large $N$, we get

$$
\frac{d \bar{K}_{N}}{d N}=\frac{1}{N}(1-2 \delta) \bar{K}_{N}+\frac{2 \beta}{N}
$$

The solution of this equation is

$$
\bar{K}_{N}=\frac{2 \beta}{2 \delta-1}+\left(\bar{K}_{1}-\frac{2 \beta}{2 \delta-1}\right) N^{1-2 \delta} .
$$

For $\delta>1 / 2$ a finite connectivity is reached,

$$
\bar{K} \equiv \bar{K}_{\infty}=\frac{2 \beta}{2 \delta-1} .
$$

The previous expression imposes the boundary condition $\delta>1 / 2$, necessary in order to obtain a well-defined limiting average connectivity. Eq. (2.5), together with the experimental estimates of $\bar{K} \sim 1.9-2.3$, allows to set a first restriction to the parameters $\beta$ and $\delta$. Imposing $\bar{K}=2$, we are led to the relation

$$
\beta=2 \delta-1 \text {. }
$$

Moreover, estimations of addition and deletion rates $\alpha$ and $\delta$ from yeast [42 give a ratio $\alpha / \delta \leq 10^{-3}$. For proteomes of size $N \sim 10^{3}$, as in the case of the yeast, 


\section{A)}

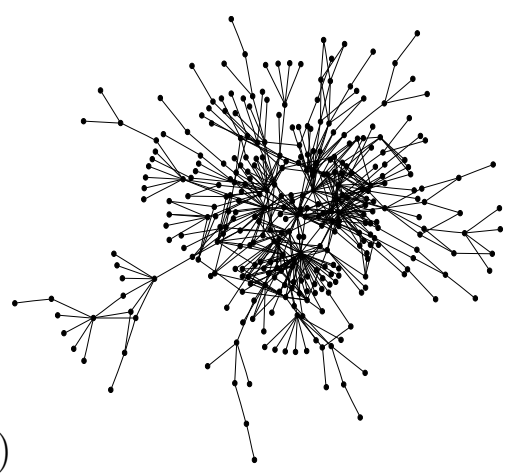

B)

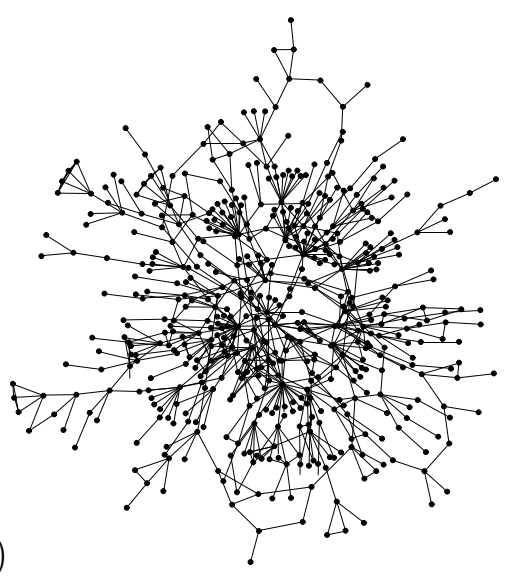

Fig. 3. A) An example of a small proteome interaction map (giant component, $\Omega_{\infty}$ ) generated by the model with $N=10^{3}, \delta=0.58$, and $\beta=0.16$. B) Real yeast proteome map obtained from the MIPS database 26]. We can observe the close similitude between the real map and the output of the model.

this leads to $\beta / \delta \leq 10^{-3} N \sim 1$. Using the safe approximation $\beta / \delta \approx 0.25$, together with the constraint (2.5), we obtain the approximate values

$$
\delta=0.58, \quad \beta=0.16 .
$$

which will be used through the rest of the paper.

Simulations of the model start form a connected ring of $N_{0}=5$ nodes, and proceed by iterating the rules until the desired network size is achieved.

\section{Results}

Computer simulations of the proposed model reproduce many of the regularities observed in the real proteome data. As an example of the output of the model, in figure 3A we show an example of the giant component $\Omega_{\infty}$ (the largest cluster of connected proteins) of a realization of the model with $N=10^{3}$ nodes. This figure clearly resembles the giant component of real yeast networks, as we can see comparing with figure $3 \mathrm{~B}$, and we can appreciate the presence of a few highly connected hubs plus many nodes with a relatively small number of connections. The size of the giant component for $N=10^{3}$, averaged of $10^{4}$ networks, is $\left|\Omega_{\infty}\right|=$ $472 \pm 87$, in good agreement with Wagner's data $\left|\Omega_{\infty}^{W}\right|=466$ for a yeast with a similar total number of proteins (the high variance in our result is due to the large fluctuations in the model for such small network size $N)$. On the other hand, in figure 4 we plot the connectivity $P(k)$ obtained for networks of size $N=10^{3}$. In this figure we observe that the resulting connectivity distribution can be fitted

${ }^{a}$ Figure kindly provided by W. Basalaj (see http://www.cl.cam.uk/ wb204/GD99/\#Mewes). 


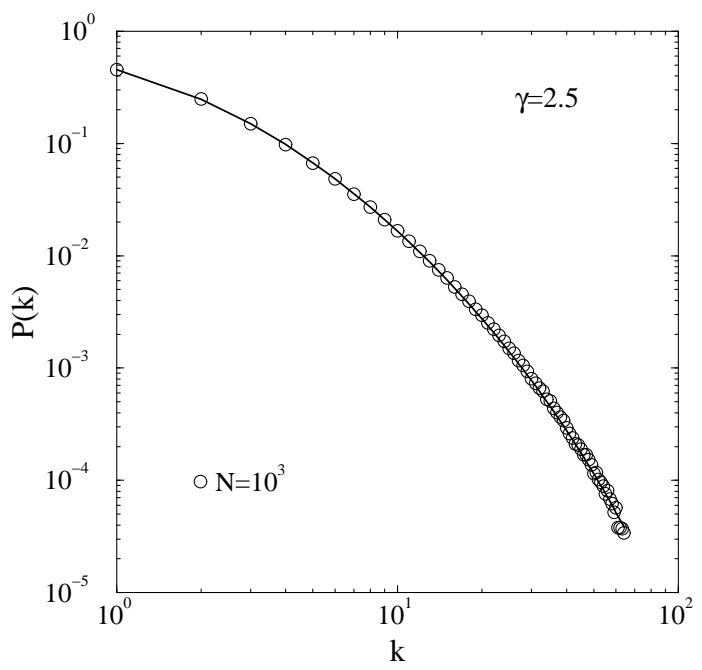

Fig. 4. Degree distribution $P(k)$ for the model, averaged over $10^{4}$ networks of size $N=10^{3}$. The distribution shows a characteristic power law behavior, with exponent $\gamma=2.5 \pm 0.1$ and an exponential cut-off $k_{c} \simeq 28$.

to a power-law win an exponential cut-off, of the form given by Eq. (1.1), with parameters $\gamma=2.5 \pm 0.1$ and $k_{c} \simeq 28$, in good agreement with the measurements reported in Refs 42] and 19].

An additional observation from Wagner's study of the yeast proteome is the presence of SW properties. We have found also similar topological features in our model, using the considered set of parameters. The proteome graph is defined by a pair $\Omega_{p}=\left(W_{p}, E_{p}\right)$, where $W_{p}=\left\{p_{i}\right\},(i=1, \ldots, N)$ is the set of $N$ proteins and $E_{p}=\left\{\left\{p_{i}, p_{j}\right\}\right\}$ is the set of edges/connections between proteins. The adjacency matrix $\xi_{i j}$ indicates that an interaction exists between proteins $p_{i}, p_{j} \in \Omega_{p}\left(\xi_{i j}=1\right)$ or that the interaction is absent $\left(\xi_{i j}=0\right)$. Two connected proteins are thus called adjacent and the degree of a given protein is the number of edges that connect it with other proteins.

The SW pattern can be detected from the analysis of two basic statistical quantities: the clustering coefficient $C_{v}$ and the average path length $L$. Let us consider the adjacency matrix and indicate by $\Gamma_{i}=\left\{p_{i} \mid \xi_{i j}=1\right\}$ the set of nearest neighbors of a protein $p_{i} \in W_{p}$. The clustering coefficient for this protein is defined as the number of connections between the proteins $p_{j} \in \Gamma_{i}$ [45]. Denoting

$$
\mathcal{L}_{i}=\sum_{j=1}^{N} \xi_{i j}\left[\sum_{k \in \Gamma_{i}} \xi_{j k}\right],
$$

we define the clustering coefficient of the $i$-th protein as

$$
c_{v}(i)=\frac{2 \mathcal{L}_{i}}{k_{i}\left(k_{i}-1\right)},
$$


Table 1. Comparison between the observed regularities in the yeast proteome 42 , the model predictions with $N=10^{3}$, $\delta=0.58$ and $\beta=0.16$, and a random network with the same size and average connectivity as the model. The quantities $X$ represent averages over the whole graph; $X^{g}$ represent averages over the giant component.

\begin{tabular}{cccc}
\hline & Yeast proteome & Network model & Random network \\
\hline $\bar{K}$ & 1.83 & $2.2 \pm 0.5$ & $2.00 \pm 0.06$ \\
$\bar{K}^{g}$ & 2.3 & $4.3 \pm 0.5$ & $2.41 \pm 0.05$ \\
$\gamma$ & 2.5 & $2.5 \pm 0.1$ & - \\
$\left|\Omega_{\infty}\right|$ & 466 & $472 \pm 87$ & $795 \pm 22$ \\
$C_{v}^{g}$ & $2.2 \times 10^{-2}$ & $1.0 \times 10^{-2}$ & $1.5 \times 10^{-3}$ \\
$L^{g}$ & 7.14 & $5.1 \pm 0.5$ & $9.0 \pm 0.4$ \\
\hline
\end{tabular}

where $k_{i}$ is the connectivity of the $i$-th protein. The clustering coefficient is defined as the average of $c_{v}(i)$ over all the proteins,

$$
C_{v}=\frac{1}{N} \sum_{i=1}^{N} c_{v}(i),
$$

and it provides a measure of the average fraction of pairs of neighbors of a node that are also neighbors of each other.

The average path length $L$ is defined as follows: Given two proteins $p_{i}, p_{j} \in W_{p}$, let $L_{\min }(i, j)$ be the minimum path length connecting these two proteins in $\Omega_{p}$. The average path length $L$ will be:

$$
L=\frac{2}{N(N-1)} \sum_{i<j}^{N} L_{m i n}(i, j)
$$

Random graphs, where nodes are randomly connected with a given probability $p$ [6], have a clustering coefficient inversely proportional to the network size, $C_{v}^{\text {rand }} \approx$ $\bar{K} / N$, and an average path length proportional to the logarithm of the network size, $L^{\text {rand }} \approx \log N / \log \bar{K}$. At the other extreme, regular lattices with only nearestneighbor connections among units are typically clustered and exhibit long average paths. Graphs with SW structure are characterized by a high clustering with $C_{v} \gg$ $C_{v}^{r a n d}$, while possessing an average path comparable with a random graph with the same connectivity and number of nodes.

In Table 1 we report the values of $\bar{K}, \gamma,\left|\Omega_{\infty}\right|, C_{v}$, and $L$ for our model, compared with the values reported for the yeast $S$. cerevisiae [19,42], and the values corresponding to a random graph with size and connectivity comparable with both the model and the real data. Except the average connectivity of the giant component, which is slightly larger for the model, all the magnitudes for the model compare quite well with the values measured for the yeast. On the other hand, the values obtained for a random graph support the conjecture of the SW properties of the protein network put forward in Ref. 442]. 


\section{Discussion}

The analysis of complex biological networks in terms of random graphs is not new. Early work suggested that the understanding of some general principles of genome organization might be the result of emergent properties within random networks of interacting units [21,22]. An important difference emerges, however, from the new results about highly heterogeneous networks: the topological organization of metabolic and protein graphs is very different from the one expected under totally random wiring and as a result of their heterogeneity, new qualitative phenomena emerge (such as the robustness against mutation). This supports the view that cellular functions are carried out by networks made up by many species of interacting molecules and that networks of interactions might be at least as important as the units themselves 15, 33].

Our study has shown that the macroscopic features exhibited by the proteome are also present in our simple model. This is surprising, since it is obvious that different proteins and protein interactions play different roles and operate under very different time scales and our model lacks such specific properties, dynamics or explicit functionality. Using estimated rates of addition and deletion of protein interactions as well as the average connectivity of the yeast proteome, we accurately reproduce the available statistical regularities exhibited by the real proteome. In this context, although data from yeast might involve several sources of bias, it has been shown that the same type of distribution is observable in other organisms, such as the protein interaction map of the human gastric pathogen Helicobacter pylori or in the p53 network (Jeong and Barabási, personal communication).

These results suggest that the global organization of protein interaction maps can be explained by means of a simple process of gene duplication plus diversification. These are indeed the mechanisms known to be operating in genome evolution (although the magnitude of the duplication event can be different). One important point to be explored by further extensions of this model is the origin of the specific parameters used. The use of evolutionary algorithms and optimization procedures might provide a consistent explanation of the particular values observed and their relevance in terms of functionality. A different source of validation of our model might be the study of proteome maps resulting from the evolution of resident genomes [3]: the genomes of endosymbionts and cellular organelles display an evolutionary degradation that somehow describe an inverse rule of proteome reduction. Reductive evolution can be almost extreme, and available data of resident proteomes might help to understand how proteome maps get simplified under the environmental conditions defined by the host genome. If highly connected nodes play a relevant role here, perhaps resident genomes shrink by loosing weakly connected nodes first.

Most of the classic literature within this area deal with the phylogenetic consequences of duplication and do not consider the underlying dynamics of interactions between genes. We can see, however, that the final topology has nontrivial con- 
sequences: this type of scale-free network will display an extraordinary robustness against random removal of nodes [i] and thus it can have a selective role. But an open question arises: is the scale-free organization observed in real proteomes a byproduct of the pattern of duplication plus rewiring (perhaps under a low-cost constraint in wiring) and thus we have "robustness for free"? The alternative is of course a fine-tuning of the process in which selection for robustness has been obtained by accepting or rejecting single changes. Further model approximations and molecular data might provide answers to these fundamental questions.

\section{Acknowledgements}

The authors thank J. Mittenthal, R. Ferrer, J. Montoya, S. Kauffman and A. Wuensche for useful discussions. This work has been supported by a grant PB97-0693 and by the Santa Fe Institute (RVS). RPS acknowledges financial support from the Ministerio de Ciencia y Tecnología (Spain).

\section{References}

[1] R. A. Albert, H. Jeong, and A.-L. Barabási. Error and attack tolerance of complex networks. Nature, 406:378-382, 2000.

[2] L. A. N. Amaral, A. Scala, M. Barthélémy, and H. E. Stanley. Classes of small-world networks. Proc. Natl. Acad. Sci. USA, 97:11149-11152, 2000.

[3] S. G. E. Andersson and C. Kurland. Reductive evolution of resident genomes. Trends Microbiol. 6: 263-268, 1998.

[4] A.-L. Barabási and R. Albert. Emergence of scaling in random networks. Science, 286:509-511, 1999.

[5] P. L. Bartel, J. A. Roecklein, D. SenGupta, and S. A. Fields. A protein linkage map of Escherichia coli bacteriohage t7. Nature Genet., 12:72-77, 1996.

[6] B. Bollobás. Random Graphs. Academic Press, London, 1985.

[7] S. Bornholdt and K. Sneppen. Robustness as an evolutionary principle. Proc. Roy. Soc. Lond. B, 267:2281-2286, 2000.

[8] D. Fell and A. Wagner. The small world of metabolism. Nature Biotech., 18:1121, 2000.

[9] R. Ferrer i Cancho, C. Janssen, and R. V. Solé. The small world of human language. Procs. Roy. Soc. London B, 268:2261-2266, 2001.

[10] R. Ferrer i Cancho, C. Janssen, and R. V. Solé. The topology of technology graphs: small world pattern in electronic circuits. Phys. Rev. E, 63:32767, 2001.

[11] R. Ferrer i Cancho and R. V. Solé. Optimization in complex networks. Phys. Rev. Lett. (submitted, 2000).

[12] S. Fields. Proteomics in genomeland. Science, 409:861-921, 2001.

[13] M. Flajolet, G. Rotondo, L. Daviet, F. Bergametti, G. Inchauspe, P. Tiollais, C. Transy, and P. Legrain. A genomic approach to the Hepatitis $\mathrm{C}$ virus generates a protein interaction map. Gene, 242:369-379, 2000.

[14] M. Fromont-Racine, J. C. Rain, and P. Legrain. Towards a functional analysis of the yeast genome through exhaustive two-hybrid screens. Nature Genet., 16:277-282, 1997.

[15] L. H. Hartwell, J. J. Hopfield, S. Leibler, and A. W. Murray. From molecular to modular cell biology. Nature, 402:C47-C52, 1999. 
[16] T. R. Hazbun and S. Fields. Networking proteins in yeast. Proc. Natl. Acad. Sci. USA, 98:4277-4278, 2001.

[17] T. Ideker, V. Thorsson, J. A. Ranish et al. Integrated genomic and proteomic analyses of a systematically perturbed metabolic network. Science 292: 929-934, 2001.

[18] T. Ito, K. Tashiro, S. Muta, R. Ozawa, T. Chiba, M. Nishizawa, K. Yamamoto, S. Kuhara, and Y. Sakaki. Toward a protein-protein interaction map of the budding yeast: A comprehensive system to examine two-hybrid interactions in all possible combinations between the yeast proteins. Proc. Natl. Acad. Sci. USA, 97:1143-1147, 2000 .

[19] H. Jeong, S. Mason, A. L. Barabási, and Z. N. Oltvai. Lethality and centrality in protein networks. Nature, 411:41, 2001.

[20] H. Jeong, B. Tombor, R. Albert, Z. N.Oltvai, and A.-L. Barabasi. The large-scale organization of metabolic networks. Nature, 407:651-654, 2001.

[21] S. A. Kauffman. Metabolic stability and epigenesis in randomly connected nets. $J$. Theor. Biol., 22:437-467, 1962.

[22] S. A. Kauffman. Origins of Order. Oxford, New York, 1993.

[23] E. S. Lander and et al. Initial sequencing and analysis of the human genome. Nature, 409:861-921, 2001.

[24] A. L. Lloyd and R. M. May. How viruses spread among computers and people. Science, 292:1316-1317, 2001.

[25] S. McCraith, T. Holtzman, B. Moss, and S. Fields. Genome-wide analysis of vaccinia virus protein-protein interactions. Proc. Natl. Acad. Sci. USA, 97:4879-4884, 2000.

[26] H. W. Mewes, K. Heumann, A. Kaps, K. Mayer, F. Pfeiffer, S. Stocker, and D. Frishman. Mips: a database for genomes and protein sequences. Nucleic Acids Res., 27:44-48, 1999.

[27] S. Ohno. Evolution by gene duplication. Springer, Berlin, 1970.

[28] C. A. Onzonnis and P. D. Karp. Global properties of the metabolic map of Escherichia coli. Genome Res. 10: 568-576, 2000.

[29] R. Pastor-Satorras, A. Vázquez, and A. Vespignani. Dynamical and correlation properties of the internet. Phys. Rev. Lett., 87:258701, 2001.

[30] R. Pastor-Satorras and A. Vespignani. Epidemic spreading in scale-free networks. Phys. Rev. Lett., 86:3200-3203, 2001.

[31] J. C. Rain, L. Selig, H. De Reuse, V. Battaglia, C. Reverdy, S. Simon, G. Lenzen, F. Petel, J. Wojcik, V. Schachter, Y. Chemama, A. S. Labigne, and P. Legrain. The protein-protein interaction map of Helicobacter pylori. Nature, 409:743, 2001.

[32] P. Ross-Macdonald, P. S. R. Coelho, T. Roemer, S. Agarwal, A. Kumar, R. Jansen, K. H. Cheung, A. Sheehan, D. Symoniatis, L. Umansky, M. Heldtman, F. K. Nelson, H. Iwasaki, K. Hager, M. Gerstein, P. Miller, G. S. Roeder, and M. Snyder. Largescale analysis of the yeast genome by transposon tagging and gene disruption. Nature, 402:413-418, 1999.

[33] R. V. Solé, I. Salazar-Ciudad, and S. A. Newman. Gene network dynamics and the evolution of development. Trends Ecol. Evol., 15:479-480, 2000.

[34] K. E. Stephan, C-C. Hilgetag, G. A. P. C. Burns, M. A. O’Neill, M. P. Young and R. Kötter. Computational analysis of functional connectivity between areas of primate cerebral cortex. Phil. Trans. Roy. Soc. Lond. B 355: 111-126, 2000.

[35] S. H. Strogatz. Exploring complex networks. Nature, 410:268-276, 2001.

[36] D. Thieffry, A. M. Huerta, E. Pérez-Rueda, and J. Collado-Vives. From specific gene regulation to genomic networks: a global analysis of transcriptional regulation in Escherichia coli. BioEssays, 20:433-440, 1998.

[37] A. Vázquez, A. Flammini, A. Maritan, and A. Vespignani. Modelling of protein 

interaction networks, 2001. cond-mat/0108043.

[38] J. C. Venter and et al. The sequence of the human genome. Science, 291:1305, 2001.

[39] B. Vogelstein, D. Lane, and A. J. Levine. Surfing the p53 network. Nature, 408:307310, 2000.

[40] A. Wagner. Evolution of gene networks by gene duplications: A mathematical model and its implications on genome organization. Proc. Natl. Acad. Sci. USA, 91:43874391, 1994.

[41] A. Wagner. Robustness against mutations in genetic networks of yeast. Nature Genet., 24: 355-361, 2000.

[42] A. Wagner. The yeast protein interaction network evolves rapidly and contains few redundant duplicate genes. Mol. Biol. Evol., 18:1283-1292, 2001.

[43] A. Wagner. and D. A. Fell. The small world inside large metabolic networks. Proc. Roy. Soc. London B 268: 1803-1810, 2001.

[44] A. J. M. Walhout, R. Sordella, X. W. Lu, J. L. Hartley, G. F. Temple, M. A. Brasch, N. Thierry-Mieg, and M. Vidal. Protein interaction mapping in c. elegans using proteins involved in vulval development. Science, 287:116-122, 2000.

[45] D. J. Watts and S. H. Strogatz. Colective dynamics of 'small-world' networks. Nature, 393:440-442, 1998.

[46] K. H. Wolfe and D. C. Shields. Molecular evidence for an ancient duplication of the entire yeast genome. Nature, 387:708-713, 1997.

[47] X. Wu, J. H. Bayle, D. Olson, and A. J. Levine. The P53 MDM-2 autoregulatory feedback loop. Gen. Dev., 7:1126, 1993. 